\title{
La integración de Competencias Transversales en la Educación Superior en Ingenierías mediante herramientas de e-learning. El caso de la ETSII en la UPV

\author{
The integration of Transversal Competences in Higher Education in Engineering \\ through e-learning tools. The case of the ETSII at the UPV
}

\author{
José Felipe Villanueva López, Mª del Mar Eva Alemany Díaz, Ana Vallés Lluch, Jorge García-Serra García, @PIME \\ jovillo0@upvnet.upv.es,mareva@omp.upv.es, avalles@ter.upv.es, jgarcias@ita.upv.es
}

Escuela Técnica Superior de Ingeniería Industrial (ETSII), Universitat Politècnica de València, España

\begin{abstract}
Resumen- Uno de los objetivos de la Educación Superior cada vez más demandado por el mercado laboral es garantizar el desarrollo de habilidades profesionales, sociales y éticas del alumnado. Compatibilizar el aprendizaje de este tipo de competencias de carácter transversal (CT) con las competencias específicas propias de la titulación en el marco temporal de los estudios, no es tarea sencilla especialmente para algunas de estas competencias y en titulaciones de perfil ingenieril. Por este motivo, en la Escuela Técnica Superior de Ingeniería Industrial (ETSII) de la Universitat Politècnica de València (UPV) se ha diseñado una plataforma e-learning por parte del profesorado, estudiantes y empleadores, coordinados por la Subdirección de Innovación Educativa y en el marco de diferentes Proyectos de Innovación y Mejora Educativa de la UPV, Esta plataforma permite al estudiantado formarse en CT de manera autónoma según sus necesidades, y al profesorado recurrir a metodologías adecuadas a sus asignaturas y nivel de dominio adaptadas al ámbito ingenieril. De esta forma, la plataforma puede usarse como eje de coordinación vertical entre asignaturas, para el desarrollo de $\mathrm{CT}$ en los distintos niveles de dominio a lo largo de los estudios del alumnado de la ETSII.
\end{abstract}

Palabras clave: competencia transversal, formación, aprendizaje activo, aprendizaje autónomo, e-learning, ingenieria

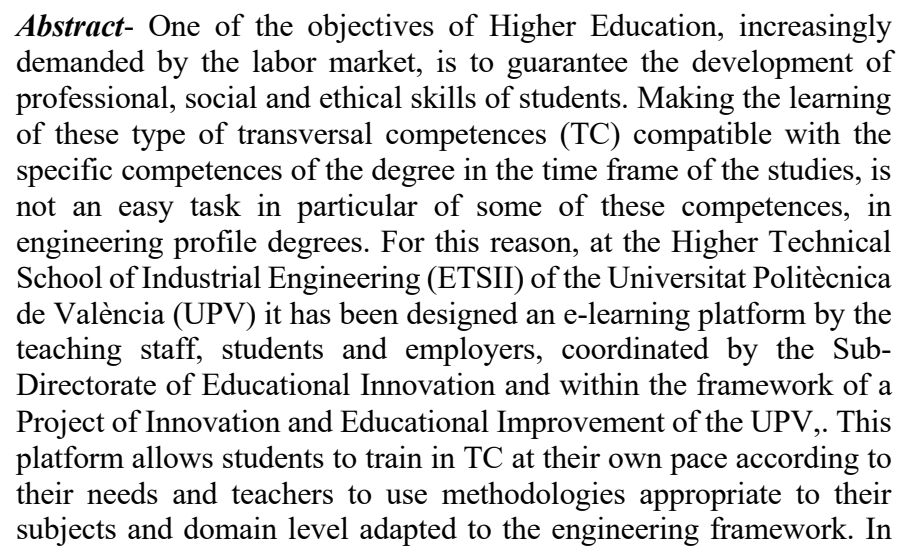

Abstract- One of the objectives of Higher Education, increasingly of these type of transversal competences (TC) compatible with the specific competences of the degree in the time frame of the studies, is not an easy task in particular of some of these competences, in engineering profile degrees. For this reason, at the Higher Technical School of Industrial Engineering (ETSII) of the Universitat Politècnica de València (UPV) it has been designed an e-learning platform by the teaching staff, students and employers, coordinated by the SubDirectorate of Educational Innovation and within the framework of a Project of Innovation and Educational Improvement of the UPV,. This platform allows students to train in TC at their own pace according to subjects and domain level adapted to the engineering framework. In this way, the platform can be used as a vertical coordination axis between subjects, for the development of TC at the different levels of mastery throughout the ETSII students' studies.

Keywords: transversal competence, training, active learning, selflearning, e-learning, engineering

\section{INTRODUCCIÓN}

El mercado laboral demanda cada vez más perfiles profesionales capaces de adaptarse con éxito y rapidez al entorno cambiante. En este contexto, uno de los principales objetivos de la Educación Superior consiste en dotar a sus estudiantes de conocimientos y competencias que les permitan afrontar con éxito su futura vida profesional (Sá y Serpa, 2018) y que garanticen al mismo tiempo el desarrollo de habilidades sociales, éticas y profesionales (Oksana, Galstyan-Sargsyan, López-Jiménez y Pérez-Sánchez, 2020).

\section{A) Sobre la necesidad de formación (también) en competencias} transversales

Por este motivo, existe cada vez una mayor conciencia de la responsabilidad de dotar a los estudiantes no sólo de una sólida formación científica y técnica, sino también de formarlos simultáneamente en competencias transversales (CT) transferibles a cualquier área de conocimiento (Szafranski , Golinski y Simi, 2017). Adicionalmente en el caso de carreras técnicas, muchas de las CT se encuentran en línea con los resultados de aprendizaje relacionados con la aplicación práctica de la ingeniería y de diseño y proyecto exigidas por las agencias internacionales de acreditación como ENAEE (European Network for Accreditation of Engineering Education) o ABET (Accreditation Board for Engineering and Technology; Baltimore, USA) (Alemany, Villanueva, VallésLluch y García-Serra, 2020).

Ante esta situación, una de las acciones más relevantes llevadas a cabo por la Universitat Politècnica de València

\footnotetext{
${ }^{1}$ Alarcón F., Alfonso D., Amigó V, Andrés J., Aparicio F., Aragonés P., Arroyo M., Bastante M.J., Bes M.A., Boza A., Castello F., Conesa P., Cuenca L., Denia F., Dols J., Espinós A., Esteso A,. Esteve R., Fernández R., Ferrer E., Fuentes J.L., Fuentes P., Fuertes-Miquel V.S, Fuster M.V., Gimenez F., Giner J., Iborra A., Lapuebla A., Lisón M.P., López P.A., López G., Lozano J.F., Maheut J.P., Marín J.A., Martín R., Martínez J.L., Martínez M., Martón I., Masip X., Mendoza J.A., Miralles C., Molina M., Mondragón A., Monfort R., Montagud C., Monzó J.M., Museros P., Palomo M.J., Pérez M., Peris M., Portillo N., Prades C., Ribes M.D., Rius G., Rodríguez M.A., Royo R., Rupérez M.J., Sahuquillo O., Salavert J.M., Sánchez E., Sánchez R., Sánchez-Moreno L., Soriano J., Tormo G., Tort I., Valera A., Vargas C., Vicente A., Vidaurre A., Vilariño G., Viñoles R., Vivas D.
} 
(UPV) para adaptarse al EEES, a las agencias nacionales e internacionales de acreditación y a las necesidades del mercado laboral, fue el Proyecto de Competencias Transversales (CT) en el marco del cual se han definido $13 \mathrm{CT}$ con dos objetivos principales:

a) Establecer una estrategia de formación y evaluación sistemática de las competencias transversales, definiendo dónde se adquieren y cómo deben ser evaluadas,

b) Acreditar la adquisición de dichas competencias.

Certificar la adquisición de las competencias no es un hecho baladí. Por este motivo, el proyecto institucional de la UPV propone tres vías para ello: las asignaturas de las propias titulaciones, el Trabajo Fin de Grado o Trabajo Fin de Máster y la realización de actividades extracurriculares (actividades de voluntariado, actividades deportivas, participación en proyectos, concursos, etc.). Además, establece tres niveles de dominio para aumentar progresivamente el grado de profundización del alumnado en cada competencia: Nivel I ( $1^{\circ}$ y $2^{\circ}$ grado); Nivel II ( $3^{\circ}$ y $4^{\circ}$ grado) y Nivel III ( $1^{\circ}$ y $2^{\circ}$ máster $)$.

Como un medio para la formación en CT, dentro de las líneas prioritarias de la UPV se han identificado el impulso de las metodologías de aprendizaje activo, como el ABP (Aprendizaje Basado en Proyectos o Problemas). Estas metodologías incluyen aquellas aproximaciones metodológicas que abarcan tanto el propio aprendizaje basado en proyectos, problemas, o casos combinados con los ingredientes necesarios del aprendizaje cooperativo para el desarrollo de competencias tanto específicas como transversales (Alemany, Vallés, Villanueva y García-Serra, 2021). Se tratan, por tanto, de metodologías complejas y cercanas a la realidad, que favorecen experiencias de aprendizaje de alto impacto educativo, la integración de conocimientos, la transferencia de lo aprendido a la realidad profesional, etc.

En este marco de actuación, es necesario que previamente se hayan trabajado y evaluado conocimientos relacionados tanto con competencias específicas (CE) como CT que posteriormente se desarrollarán, ampliarán e integrarán conjuntamente, empleando metodologías de aprendizaje activo. Es por ello que estas metodologías de aprendizaje activo no son incompatibles, sino más bien deben ser complementadas con actividades que permitan el conocimiento y formación en CT y $\mathrm{CE}$ de manera integral. Por tanto, es necesario seguir formando a los estudiantes no sólo en CE sino también en dichas CT de manera conjunta. De hecho, y como bien apunta Cano (2008), uno de los tres rasgos que caracterizan a las competencias es que también se aprenden: el hecho de poseer de forma innata ciertas inteligencias es un buen punto de partida, pero no garantiza ser competente. Las competencias deben desarrollarse con formación inicial, con formación permanente $\mathrm{y}$ con experiencia a lo largo de la vida. Es decir, no es suficiente con que el profesorado ponga el marco de trabajo en sus asignaturas para que el alumnado desarrolle o potencie sus CT, sino que es necesaria también la formación, a través de metodologías con complejidad creciente, en este tipo de competencias para potenciar un dominio adecuado de la mismas que les garantice el éxito académico y profesional.

B) No todas las competencias transversales son iguales... algunas son "difíciles"

Las Escuelas y Facultades de la UPV están realizando una importante labor de coordinación para asegurar que todas las competencias se trabajan en los diferentes niveles de dominio a través de tres vías: asignaturas, Trabajos Fin de Grado y de Máster y actividades extracurriculares. Sin embargo, la experiencia acumulada durante estos años muestra claramente que la formación y evaluación no es percibida por el profesorado con el mismo nivel de complejidad para todas las CT. En concreto, por parte de la Escuela Técnica Superior de ingenieros Industriales (ETSII) de la UPV se ha apreciado que 6 de las 13 CT de la UPV plantean serias dificultades ya no solo desde el punto de vista del docente sino también del estudiantado que las ha de trabajar. Esta conclusión se deriva de las reuniones de seguimiento por parte de las comisiones académicas de los distintos títulos tras la implantación del sistema de CT en la UPV. El hecho de singularizarlas denominándolas "difíciles" pretende poner de manifiesto la mayor complejidad encontrada al trabajarlas y evaluarlas en el contexto de las asignaturas del ámbito ingenieril, sin depreciar las dificultades que impone, y el esfuerzo a realizar, para trabajar y evaluar las otras CT, con grupos de alumnos en muchas ocasiones bastante numerosos. Por otro lado, estas CT están entre las más valoradas por las empresas españolas en los/as Ingenieros/as (Instituto de la Ingeniería de EspañaAsociación para el Progreso de la Dirección, 2017). Estas CT "difíciles" son: Innovación, creatividad y emprendimiento; Trabajo en equipo y liderazgo; Responsabilidad ética, medioambiental y profesional; Conocimiento de problemas contemporáneos; Aprendizaje permanente, y Planificación y gestión del tiempo.

Ante la carencia de soporte, especialmente para los estudiantes y más aún desde un punto de vista ingenieril, desde la Subdirección de Innovación Educativa de la ETSII de la UPV se propuso como objetivo facilitar la adquisición y evaluación de dichas competencias con un modelo eficaz que no supusiera una carga adicional para el profesorado, a través del desarrollo de una plataforma de e-learning. Para ello, se lanzaron tres proyectos de innovación y mejora educativa (PIME/19-20/150, 151 y 152) con el objetivo de desarrollar material e-learning en las seis CT anteriormente mencionadas, Se ha estado trabajando al respecto durante más de 2 años con la colaboración de más de 70 participantes entre profesores, estudiantes, egresados y empleadores. El resultado es una plataforma de e-learning con recursos genéricos y específicos orientados al ámbito ingenieril que soporta, facilita y permite la integración de las CT con las CE. Adicionalmente, dicha plataforma e-learning está orientada a conseguir la coordinación metodológica en la formación y evaluación de dichas $\mathrm{CT}$, puesto que se encuentra estructurada por niveles de dominio, indicando las metodologías más convenientes según el curso en el que se encuentre el estudiante.

Así, el presente artículo tiene como objetivo mostrar la plataforma e-learning en las seis CT difíciles y la utilidad de la misma a la hora de formar y evaluar a los estudiantes en el ámbito de la educación superior y más concretamente de las ingenierías. El resto del artículo se encuentra estructurado de la siguiente manera. En la sección 2 se establece el punto de partida sobre el que se construyó la actual plataforma de $e$ learning y cuyo proceso de elaboración se describe en la sección 3. La sección 4 describe la plataforma de e-learning resultante así como sus diferentes formas de uso. Finalmente, en la sección 5 se establecen las conclusiones y futuras líneas de trabajo.

\section{CONTEXTO}


Las metodologías de aprendizaje activo están cobrando una importancia cada vez mayor como una forma de trabajar simultáneamente las CT y CE de las titulaciones y como un medio para que los alumnos se enfrenten a situaciones cercanas a la realidad profesional La implantación de estas metodologías casi con toda seguridad requerirá una redistribución de créditos de teoría de aula y seminario e incluso de revisión de contenidos, lo que podría requerir compaginar el ABP con la metodología de docencia inversa. La docencia inversa, a su vez necesitará de la elaboración de material para el trabajo autónomo por parte del estudiante que puede ser material escrito o audiovisual a través de la red (e-learning) tanto de conocimientos relacionados con $\mathrm{CE}$ como también con $\mathrm{CT}$. Rodrigues, Almeida, Figueiredo y Lopes (2019) definen el $e$ learning como un sistema innovador basado en la web sustentada en tecnologías digitales y otras formas de materiales educativos que apoyan y mejoran los procesos de aprendizaje. Las ventajas de los sistemas de e-learning son que proporciona oportunidades de aprendizaje sin las típicas restricciones de lugar y tiempo, y apoya nuevos enfoques de enseñanza y aprendizaje que implican una mezcla de métodos de aprendizaje tradicionales y e-learning (Alhabeeb y Rowley, 2018).

Aunque la UPV dispone de una web de CT y de material para profesores en su plataforma de teleformación, no hay un espacio dedicado a la formación del alumnado. Por otro lado, tampoco se conoce ninguna iniciativa que utilice un sistema e-learning como eje de la coordinación horizontal $\mathrm{y}$, especialmente, vertical entre las asignaturas de un título.

Con la intención de contribuir a cubrir el vacío detectado, desde la Subdirección de Innovación Educativa de la ETSII, se llevó a cabo como una primera aproximación un acopio de material disponible en otras webs y en el repositorio institucional de la UPV, que pudiera servir como punto de partida para este proyecto. La primera versión de la web de formación on-line en $\mathrm{CT}$ difíciles, se nutrió sobre todo de material de las webs de otras Universidades (videos, textos, cuestionarios, enlaces, etc.), como pueden ser la University of Edinburgh, Sussex, Kent, o la UNSW Sydney, aunque también de videos de acceso libre a través de YouTube y videos Media UPV, así como enlaces a textos de profundización en estas CT. Los objetos de aprendizaje y evaluación contenidos en la web resultaron ya de por sí de enorme valor tanto para los estudiantes como para los profesores implicados en estas CT, siendo, pues, el punto de partida del Proyecto que aquí se presenta (Fig. 1).

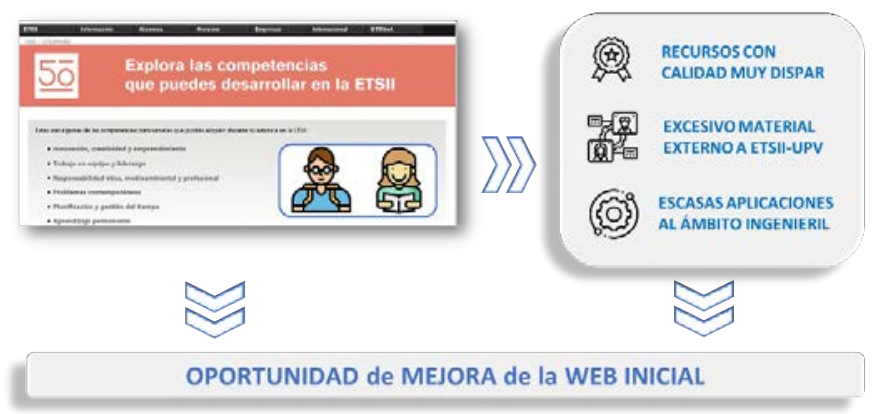

Figura 1. Punto de partida: análisis de webs de apoyo inicial en $C T$ dificiles

A modo de ejemplo, la versión original de la web para la CT de Innovación, creatividad y emprendimiento se puede consultar en la dirección web de la ETSII (2019). La estructura y enfoque adoptado para el desarrollo de esta web se encuentra descrita con detalle en Alemany et al. (2021). Si bien estas webs de apoyo a las seis CT denominadas difíciles supusieron un avance sustancial en la dirección correcta, se identificaron ciertos inconvenientes en el empleo de este material "externo":

i) El material no se encontraba estructurado por niveles de dominio, como sería deseable para que el estudiantado accediera a estos contenidos.

ii) Se planteaban ejemplos o casos concretos, que en la mayoría de los casos no se refieren a aplicaciones ingenieriles, como sería deseable en vistas a integrar la formación en estas competencias con la formación en competencias específicas de Graduados o Másteres en Ingenierías.

iii) Los recursos eran bastante heterogéneos: convivían videos muy visuales y amenos con otros videos o textos bastante más arduos, y en su mayoría en inglés.

iv) Las experiencias concretas de estudiantes contenidas en esta versión inicial de las webs se entienden bien en el contexto de otras Universidades, pero pueden resultar algo ajenas a nuestros estudiantes y no verse reflejados en ellas.

En definitiva, esta primera aproximación mostraba una variedad de recursos de calidad dispar y con una fuerte presencia de material externo. Por otro lado, tenían en muchos casos poco trasfondo ingenieril y, en consecuencia, escasa capacidad de penetración en el alumnado objetivo, identificándose por tanto una gran oportunidad de mejora. Por este motivo, se propusieron tres proyectos de innovación y mejora educativa de carácter institucional (PIME/19-20/150, 151 y 152) que recogían el espíritu mencionado, trabajando cada uno de ellos dos de las anteriores CT difíciles. Para aprovechar esta oportunidad de mejora se pensó que una parte fundamental debería consistir en la participación del profesorado que debía trabajar las competencias, el estudiantado hacia quienes se dirigía el proyecto, y de los empleadores que son los que mayormente solicitan este tipo de competencias en su plantilla

El principal objetivo de los PIME planteados consistió en seguir avanzando en el desarrollo de la web, que servirá de hilo conductor para la coordinación horizontal y especialmente vertical en las asignaturas en lo que se refiere a las metodologías formativas y de evaluación de las CT difíciles. Se planteó estructurar el material por niveles de dominio, con ejemplos y casos prácticos ingenieriles, cercanos y motivadores para los estudiantes, con la implicación del profesorado y estudiantado de la ETSII. La siguiente sección muestra el proceso llevado a cabo hasta llegar a la plataforma e-learning.

\section{DESCRIPCIÓN DE LA METODOLOGÍA PARA LA CREACIÓN DE LA PlATAFORMA E-LEARNING DE CT}

Los tres proyectos de innovación y mejora educativa de carácter institucional (PIME/19-20/150, 151 y 152) se plantearon como un medio para conseguir los siguientes objetivos generales:

OG1. Contribuir a la adquisición por parte de los estudiantes de seis de las CT más valoradas por las empresas españolas en los/as Ingenieros/as percibidas, además, por el profesorado como difíciles, mejorando así la inserción laboral de los egresados y facilitando la labor del profesorado.

OG2. Definir una propuesta metodológica para la formación y evaluación de las seis CT "difíciles", basada en la coordinación por niveles de dominio a través de la creación de 
recursos web como elemento pivote estructurados como plataforma de e-learning, mediante la ampliación y mejora de los recursos web existentes, dotándole de un marcado carácter ingenieril.

El plan de trabajo para alcanzar los anteriores objetivos generales fue similar para todas las CT. A continuación, se detallan las diferentes etapas con sus respectivas fases, cuya ejecución se planteó para una duración dos años.

Etapa I) Incorporación a los PIME de los agentes interesados

Fase 1: Reunir la participación de los agentes implicados en los diferentes títulos de la ETSII

En esta fase previa, se buscó la participación del mayor número de agentes implicados en la formación y evaluación de las CT, de manera que quedasen cubiertos el máximo número posible de títulos de entre los 5 Grados y 7 Másteres que actualmente se imparten en la ETSII. Los objetivos perseguidos fueron:

1. Conseguir la mayor participación posible por parte del profesorado, en especial de aquel que trabaja y/o evalúa las CT difíciles, de sus estudiantes, así como de empleadores de egresados de los títulos de la ETSII.

2. Conseguir la mayor variedad posible de titulaciones y niveles de dominio que maximizase el alcance del público objetivo.

Para ello, la propuesta de los tres PIME anteriormente mencionados constituyó un fuerte estímulo, ya que se consiguió incrementar el sentido de vinculación y pertenencia por parte de los agentes implicados y la generación de resultados tangibles y útiles tanto para estudiantes como para el profesorado.

Etapa II) Análisis de las webs de CT-ETSII iniciales y propuestas de mejora

Esta etapa tuvo como principal objetivo el análisis de las webs originales por parte del profesorado implicado, y también de determinados estudiantes, como un primer paso para la mejora. Para ello, se diseñó un cuestionario a cumplimentar por los diferentes agentes que evaluaba tanto aspectos técnicos como de contenido de las webs de CT iniciales. A partir de este, se extrajeron conclusiones relevantes para la mejora de las mismas. Para conseguirlo, se establecieron las siguientes actividades:

3. Revisión de las webs originales para las seis CT difíciles por parte de profesores y grupos de estudiantes.

4. Identificación de carencias/oportunidades de mejora de los recursos existentes en las webs, identificando puntos fuertes y débiles.

5. Propuesta por parte de los profesores de cada asignatura de su potencial contribución a la mejora de las webs originales, e identificación de actividades/metodologías bien de las webs originales o de otras fuentes más idóneas para sus asignaturas.

6. Identificación por parte de los responsables de los PIME de CT-ETSII de posibles aportaciones desde el mundo empresarial que completasen el punto de vista práctico de las competencias.

Fase 2: Coordinación y asignación de los recursos a diseñar por cada agente implicado

Partiendo de las respuestas obtenidas al cuestionario planteado en la fase anterior, y mediante un proceso de entrevistas de los responsables de los tres PIME de CT -ETSII con los agentes implicados por asignaturas y/o CT, se procedió a definir y detallar las diferentes aportaciones a realizar por cada agente o grupo de agentes: videos profesores, videos estudiantes, videos egresados, videos externos, webs con material útil para la formación y evaluación en $\mathrm{CT}$, fichas de buenas prácticas con ejemplo de estudiantes, rúbricas, nuevas metodologías, etc. Para llevar a cabo esta asignación, el equipo de Innovación Educativa de la ETSII trabajó en la asignación de actividades para que se realizase una exploración amplia de todo el espectro posible de actividades, metodologías y niveles de dominio posibles, de tal forma que hubiera una representación variada de cada una de ellas. Una vez coordinado, se procedió a asignar tareas específicas a cada uno de los participantes en función de su disponibilidad ofreciendo siempre el apoyo requerido por parte del equipo de innovación docente. Tras el periodo de identificación, se procedió al de asignación de tareas concretas que centrasen la labor de cada participante. Las actividades llevadas a cabo se pueden resumir en:

7. Análisis por parte los responsables de los tres PIME de las respuestas obtenidas al cuestionario lanzado en la Etapa II.

8. Coordinación de actividades/metodologías entre asignaturas por niveles de dominio y de las potenciales contribuciones a la mejora de la web.

9. Asignación de las contribuciones de cada agente a las diferentes actividades/metodologías y del recurso web a mejorar.

\section{Etapa III) Diseño de la plataforma e-learning en CT difíciles}

\section{Fase 3: Diseño de las aportaciones de cada agente}

Tras la asignación de la aportación a realizar para cada agente, se pasó a la fase de diseño de cada una de ellas. Cualquier aplicación debía documentase detalladamente en su aspecto formal para su posible seguimiento por parte de otros estudiantes/profesores. Las tareas consistieron en:

10. Elaboración de la aportación o recurso web asignado a cada agente.

11. Diseñar un ejemplo de aplicación y documentar la actividad/metodología de integración de CT y CE llevada a cabo, de manera que fuera replicable por otros profesores.

Fase 4: Definición de la estructura de la plataforma $e$ learning en CT difíciles e integración de las aportaciones

Durante esta fase, se procedió a rediseñar las webs existentes con el objetivo de convertirlas en una verdadera plataforma de e-learning en CT “difíciles”. Para ello, fue necesario el rediseño de las webs iniciales integrándolas en una única web estructurando las diferentes aportaciones por niveles de dominio. Así, la principal tarea de esta fase consistió en:

12. Rediseñar las webs de CT originales con las aportaciones recibidas y las sugerencias de mejora recabadas con el cuestionario de la Etapa I.

\section{Resultado: Plataforma E-Learning CT ETSII}

Como resultado del anterior proceso, se obtuvo una única plataforma e-learning para las seis CT denominadas "difíciles" (accesible en el Blog de Competencias transversales ETSII, 2020): un espacio más moderno, simplificado y amigable en el que se encuentran integrados y estructurados de manera similar 
diversos objetos de aprendizaje orientados a cada una de las CT difíciles. En esta plataforma se han incorporado nuevas aportaciones, aunque también se han mantenido recursos ya existentes, así como ejemplos variados de aplicación consistentes fundamentalmente en vídeos sobre metodologías y su aplicación, vídeos testimoniales de diversos agentes, cuestionarios, actividades de auto-evaluación, actividades realizadas en asignaturas de la ETSII para la formación y evaluación de estas CT, webs de soporte y otros objetos de aprendizaje.

La nueva plataforma se estructura en diversas páginas. La primera de ellas, denominada de "Bienvenida" está pensada para los alumnos de nuevo ingreso para los que las CT son un concepto totalmente desconocido. Dicha página se caracteriza por una portada que define las competencias transversales tal y como se entienden en la UPV y las valoriza en el currículo del alumnado tanto a nivel académico como personal y/o laboral, con ejemplos sobre la relevancia que dichas CT tienen para algunas empresas de referencia en su sector (Fig. 2).

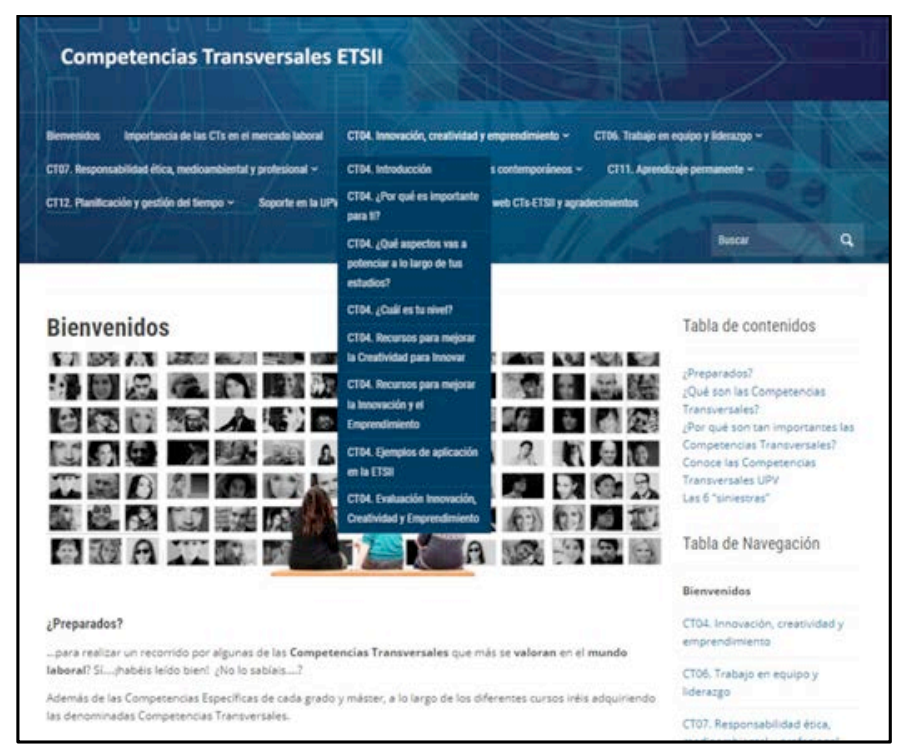

Figura 2. Página de Bienvenida de la plataforma e-learning de CT ETSII-UPV. En el menú desplegable se muestra la estructura por páginas de cada una de las CT.

Posteriormente, se incluye una página sobre la "Importancia de las CT en el mercado laboral" con testimonios de diferentes Directivos de empresas de referencia en su sector para valorizar las CT y la mayoría de los cuales han sido egresados de la ETSII. A continuación, la plataforma dedica una página a cada una de las seis CT que debido a su dificultad o complejidad a la hora de trabajar se denominan en tono coloquial como las "seis siniestras": Innovación, creatividad y emprendimiento; Trabajo en equipo y liderazgo; Responsabilidad ética, profesional y medioambiental; Conocimiento de problemas contemporáneos; Aprendizaje permanente y Planificación y Gestión del tiempo.

El desarrollo de estas competencias mantiene una estructura común y consensuada que cubre diferentes conceptos y ámbitos a través de los siguientes apartados (Fig. 3):

- Introducción: donde se define la CT en cuestión.

- ¿Por qué es importante para ti?: donde se pretende hacer comprender al estudiante la importancia de formarse también en estas CT, para lo que se incluyen, en ciertos casos, objetos de aprendizaje realizados por estudiantes, egresados y/o empleadores.
- ¿Qué aspectos vas a potenciar a lo largo de tus estudios?: en la que se incluyen los resultados del aprendizaje que se espera que los estudiantes adquieran en los tres niveles conforme vayan progresando en los diferentes cursos del título.

- ¿Cuál es tu nivel?: en la que se muestran herramientas de autoevaluación que pueden ayudar a los estudiantes a tener una idea preliminar sobre su nivel de dominio actual en la CT correspondiente.

- Recursos para mejorar: donde se incluyen diferentes objetos de aprendizaje sobre metodologías y herramientas, organizados por niveles de dominio para que los estudiantes mejoren la $\mathrm{CT}$ en cuestión. Estos objetos combinan cierto material externo, pero mayoritariamente propio de la UPV y realizado en el marco de los tres PIME de CT.

- Ejemplos de aplicación en la ETSII: donde se muestran las actividades llevadas a cabo por diferentes asignaturas en la ETSII a través de vídeos y fichas de buenas prácticas, organizadas por niveles de dominio, títulos $\mathrm{y}$ asignaturas.

- Evaluación de la CT: donde se indica al estudiante que la evaluación de cada CT la puede llevar a cabo el/la profesor/a, el/la alumno/a (auto-evaluación), o los/as compañeros/as (co-evaluación), y se muestran diversos instrumentos y ejemplos en asignaturas de la ETSII, algunos de los cuales utilizan rúbricas.

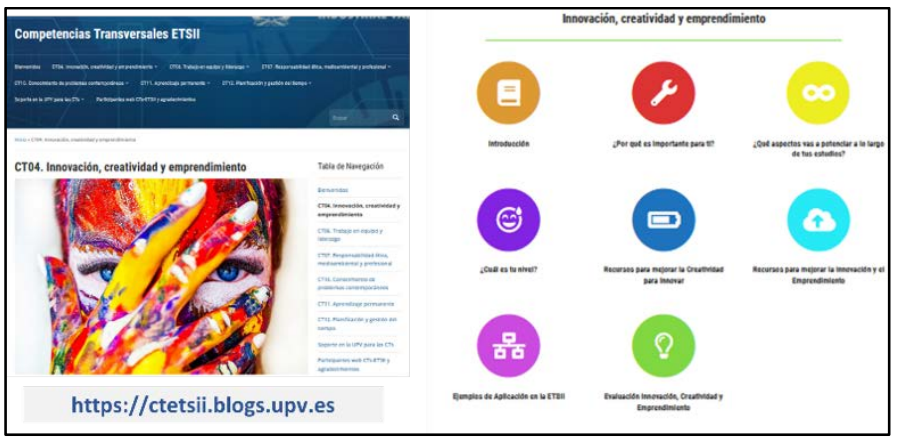

Figura 3. Estructura común de material disponible acerca de cada una de las CT "dificiles".

Como ejemplo, y para dar idea de la dimensión del proyecto y de la cantidad de recursos propios de personal de la ETSII, la sección CT04. Innovación, creatividad y emprendimiento cuenta con un vídeo de un alumno de la ETSII y dos de exalumnos emprendedores contando su experiencia al respecto de esta CT, dos test o ejercicios de autoevaluación sobre el nivel de desarrollo -uno de ellos propio-, 28 vídeos con técnicas y recursos para mejorar -12 de ellos propios-, y 11 ejemplos de aplicación (buenas prácticas) -todos ellos propios-.

La plataforma e-learning obtenida para las seis CT difíciles ofrece la posibilidad de ser utilizada de múltiples maneras. Una de ellas es que los estudiantes la utilicen de forma totalmente autónoma, lo que les permite ampliar el acceso a diferentes tipos de recursos educativos según el nivel en el que se encuentren. Se trataría de un aprendizaje auto-organizado, entendido como un método de aprendizaje que los estudiantes emprenden de forma independiente, sin seguir los requisitos curriculares prescritos o sin depender del profesorado (Ponti, 2014). Además, la plataforma de e-learning ofrece valiosos recursos y objetos de aprendizaje al estudiantado para poner en práctica estas CT a lo largo de las tareas asignadas en el curso, $\mathrm{y}$ ayuda al profesorado a formar $\mathrm{y}$ evaluar en las correspondientes CT. Se trata de que los profesores dirijan a sus alumnos a objetos y secciones concretas de la plataforma para que se formen y trabajen de manera autónoma previamente a 
las clases donde se aplicarán de manera integrada con las CE, bien a través de actividades en el aula y/o trabajos, con el ahorro sustancial en tiempo que esto conlleva. Otra alternativa es que los profesores utilicen los recursos de la plataforma para nivelar las capacidades de sus estudiantes al inicio del curso académico (Cuenca et al., 2018). Por último, la plataforma se ha concebido también como herramienta para la coordinación vertical de metodologías entre asignaturas de una misma titulación por niveles de dominio. De hecho, esta es una de las líneas de trabajo actuales en la ETSII de la UPV.

\section{CONCLUSIONES Y FUTURAS LÍNEAS DE INVESTIGACIÓN}

En este artículo se ha descrito el trabajo desarrollado durante los últimos tres años, primero por el equipo de Innovación Educativa de la ETSII y luego por parte de más de 70 agentes vinculados a dicha escuela bajo el amparo de la Dirección de la ETSII, el Vicerrectorado de Estudios, Calidad y Acreditación (VECA) y el Instituto de Ciencias de la Educación (ICE) de la UPV. A raíz de la identificación de la dificultad detectada sobre la manera de trabajar y evaluar determinadas CT de forma integrada con las $\mathrm{CE}$ en las asignaturas punto de control de las CT, el equipo de Innovación Educativa de la ETSII inició un proyecto, primero propio, y después bajo el formato de tres Proyecto de Innovación y Mejora Educativa que cubrían las seis CT difíciles. Con estos proyectos se perseguía dar soporte a los estudiantes y a los docentes a la hora de formar, trabajar y evaluar dichas CT, de manera que se minimizasen recursos docentes ya comprometidos en el aula, destinándolos a un aprendizaje más profundo de puesta en práctica.

Los recursos generados han sido de gran variedad en cuanto a asignaturas de diferentes niveles de dominio y títulos de la ETSII. Además de la plataforma de e-learning en sí misma, se ha profundizado en aportarle contenidos de acuerdo a los objetivos perseguidos, consiguiendo una plataforma de autoaprendizaje con una estructura consistente, "user friendly" con un hilo conductor que facilita la comprensión, y adquisición de las competencias a través de diferentes propuestas que las trabajan, y de una variedad de ejemplos que permiten ilustrar su aplicación en varios contextos. A través de esta plataforma se brinda apoyo a los estudiantes que, de manera independiente o siguiendo las indicaciones del profesorado, deseen formarse en estas CT de forma más aplicada, cercana a la Ingeniería y con ejemplos más alineados con su futuro trabajo profesional.

Tras una primera etapa de diseño e implementación, el equipo de trabajo se encuentra ahora evaluando los resultados en términos de consecución de mejora en el dominio de las CT citadas y de satisfacción con los productos generados. Durante este proceso se está investigando mediante un estudio pre y post utilización de la plataforma para observar posibles cambios en la percepción y asimilación por parte de los estudiantes del trabajo realizado en el desarrollo de estas CT. Por el momento, los estudiantes han acogido la iniciativa de manera positiva y el hecho de participar en la realización de vídeos y objetos de aprendizaje ha constituido un elemento motivador para ellos. Finalmente, resta por llevar a cabo un análisis global y por asignatura para poder extraer conclusiones acerca del impacto que la plataforma de e-learning ha tenido sobre el nivel competencial de los estudiantes.

\section{AgRADECIMIENTOS}

Este trabajo ha sido desarrollado en el marco de los proyectos "Coordinación metodológica a través de webs de apoyo en títulos ETSII para diferentes CT" de la convocatoria de Proyectos de Innovación y Mejora Educativa Institucionales con código PIME/19-20 Ref.150, Ref.151 y Ref.152, impulsada por el Vicerrectorado de Estudios, Calidad y Acreditación y el Instituto de Ciencias de la Educación de la UPV.

\section{REFERENCIAS}

Alemany M. M. E., Villanueva J. F., Vallés-Lluch A. y GarcíaSerra J. (2020). Web based on e-learning objects as support to the development of transversal competences for engineering students. INTED2020 Proceedings, pp. 2227-2235.

Alemany M. M. E., Vallés A., Villanueva J. F. y García-Serra J. (2021). E-learning in "innovation, creativity and entrepreneurship": Exploring the new opportunities and challenges of technologies. Journal of Small Business Strategy, 31(1), 39-50.

Alhabeeb A. y Rowley, J. (2018). E-learning critical suc $\neg$ cess factors: Comparing perspectives from academic staff and students. Computers and Education, 127, 1-12.

Cano M. E. (2008). La evaluación por competencias en la educación superior. Profesorado. Revista de currículum y formación del profesorado,12, 1-16.

Escuela Técnica Superior de Ingeniería Industrial (2019). Web para la CT de Innovación, creatividad y emprendimiento. Disponible https://www.etsii.upv.es/competencias/innovacion.php.

Escuela Técnica Superior de Ingeniería Industrial (2020). Blog de Competencias transversales ETSII. Disponible en https://ctetsii.blogs.upv.es.

Instituto de la Ingeniería de España-Asociación para el Progreso de la Dirección. (2017) Informe sobre competencias adicionales requeridas a los ingenieros por las empresas españolas. Disponible en: https://www.iies.es/singlepost/2018/02/20/APD-IIE-encuestacompetenciasingenieros

Oksana P., Galstyan-Sargsyan R., López-Jiménez P. A. y Pérez-Sánchez M. (2020) Transversal Competences in Engineering Degrees: Integrating Content and Foreign Language Teaching. Education Sciences, 10(11): 296. doi: 10.3390/educsci1011029.

Rodrigues, H., Almeida, F., Figueiredo, V y Lopes, S. L. (2019). Tracking e-learning through published papers: A systematic review. Computers and Education, 136, 8798. doi: 10.1016/j.compedu.2019.03.007.

Sá M. J. y Serpa S. (2018) Transversal Competences: Their Importance and Learning Processes by Higher Education Students. Education Sciences, 8(3):126. doi: 10.3390/educsci8030126.

Szafranski, M., Golinski, M. y Simi, H. (2017) The Acceleration of Development of Transversal Competences. Centria University of Applied Sciences: Kokkola, Finland, ISBN 978-952-7173-26-8.

Universitat Politècnica de València. (2015). Proyecto institucional de competencias transversales. https://www.upv.es/entidades/ICE/info/Proyecto_Instituc ional_CT.pdf 